\author{
A. Ashyralyev ${ }^{1,2,3}$, Y. Sozen ${ }^{4}$, F. Hezenci ${ }^{5}$ \\ ${ }^{1}$ Department of Mathematics, Near East University, Nicosia, TRNC, Mersin 10, Turkey \\ ${ }^{2}$ Peoples' Friendship University of Russia (RUDN University), 6 Miklukho-Maklaya St, \\ Moscow 117198 Russian Federation \\ ${ }^{3}$ Institute of Mathematics and Mathematical Modeling, 050010, Almaty, Kazakhstan \\ ${ }^{4}$ Department of Mathematics, Hacettepe University, 06800 Beytepe, Ankara, Turkey \\ ${ }^{5}$ Department of Mathematics, Duzce University, 81620 Konuralp, Duzce, Turkey \\ (E-mail: allaberen.ashyralyev@neu.edu.tr,ysozen@hacettepe.edu.tr,fatihhezenci@duzce.edu.tr)
}

\title{
A remark on elliptic differential equations on manifold
}

\begin{abstract}
For elliptic boundary value problems of nonlocal type in Euclidean space, the well posedness has been studied by several authors and it has been well understood. On the other hand, such kind of problems on manifolds have not been studied yet. Present article considers differential equations on smooth closed manifolds. It establishes the well posedness of nonlocal boundary value problems of elliptic type, namely Neumann-Bitsadze-Samarskii type nonlocal boundary value problem on manifolds and also DirichletBitsadze-Samarskii type nonlocal boundary value problem on manifolds, in Hölder spaces. In addition, in various Hölder norms, it establishes new coercivity inequalities for solutions of such elliptic nonlocal type boundary value problems on smooth manifolds.
\end{abstract}

Keywords: differential equations on manifolds, well-posedness, self-adjoint positive definite operator.

\section{Introduction}

In the study of partial differential equations, the importance of the well-posedness (coercivity inequalities) is well known (see, for example [1-3]). Many researchers has been studied extensively the well-posedness of nonlocal boundary value problems of elliptic type partial differential equations in the Euclidean space, which is a flat manifold, (see, e.g. [4-18] and the references therein).

In the present article, we consider differential equations on smooth closed manifolds. We establish the well-posedness of nonlocal boundary value problems Hölder spaces. Furthermore, in various Hölder norms we establish new coercivity estimates for the solutions of such boundary value problems for elliptic equations.

\section{Preliminaries}

This section provides the basic definitions and fact about the Laplacian on Riemannian manifolds. The reader is referred to $[19,20]$ and the references therein for more information and unexplained subjects.

A Riemannian manifold is a pair $(\mathcal{M}, g)$, where $\mathcal{M}$ is a smooth manifold and to each $x \in \mathcal{M}$ $\langle\cdot, \cdot\rangle_{g(x)}: T_{x} \mathcal{M} \times T_{x} \mathcal{M} \rightarrow \mathbb{R}$ is a positive definite symmetric non-degenerate bilinear form such that for all smooth vector fields $X, Y \in \Gamma_{C^{\infty}}(T \mathcal{M}), x \longmapsto\langle X(x), Y(x)\rangle_{g(x)}$ is smooth.

In the local coordinates $\left(x_{1}, \ldots, x_{n}\right),\left\{\left(\frac{\partial}{\partial x^{1}}\right)_{x}, \ldots,\left(\frac{\partial}{\partial x^{n}}\right)_{x}\right\}$ is the corresponding basis of tangent space $T_{x} \mathcal{M}, g_{i j}=\left\langle\left(\frac{\partial}{\partial x^{i}}\right)_{x},\left(\frac{\partial}{\partial x^{j}}\right)_{x}\right\rangle_{g(x)}$, and $g^{i j}$ are the entries of the inverse matrix of $\left(g_{i j}\right)$.

$\nabla_{g}: \mathscr{C}^{\infty}(\mathcal{M}) \rightarrow \Gamma_{\mathscr{C} \infty}(T \mathcal{M})$ is the gradient operator defined by

$$
\left\langle\nabla_{g} \varphi, X\right\rangle_{g}=d \varphi(X)
$$


for every $\varphi \in \mathscr{C}^{\infty}(\mathcal{M}), X \in \Gamma_{\mathscr{C} \infty}(T \mathcal{M})$. In local coordinates $\left(x_{1}, \ldots, x_{n}\right)$, the gradient $\nabla_{g} \varphi$ is equal to

$$
\sum_{i, j=1}^{n} g^{i j} \frac{\partial \varphi}{\partial x_{i}} \frac{\partial}{\partial x_{j}}
$$

From the fact $d(\varphi+\psi)=d \varphi+d \psi$ for every $\varphi, \psi \in \mathscr{C}^{1}(\mathcal{M})$ it follows that $\nabla_{g}(\varphi+\psi)=\nabla_{g} \varphi+\nabla_{g} \psi$. The fact that $d(\varphi \cdot \psi)=\varphi \cdot d \psi+\psi \cdot d \varphi$ results $\nabla_{g}(\varphi \cdot \psi)=\varphi \cdot \nabla_{g} \psi+\psi \cdot \nabla_{g} \varphi$.

If $\omega \in \Omega^{n}(\mathcal{M})$ is an $n$-form and $X$ is a vector field on $\mathcal{M}$, then $\iota_{X} \omega \in \Omega^{n-1}(\mathcal{M})$ is the $(n-1)$-form defined by

$$
\iota_{X} \omega\left(X_{1}, \ldots, X_{n-1}\right)=\omega\left(X, X_{1}, \ldots, X_{n-1}\right) .
$$

Here, $X_{1}, \ldots, X_{n-1}$ are vector fields on the Riemaniann manifold $\mathcal{M}$. From the fact that $d\left(\iota_{X} \omega\right) \in$ $\in \Omega^{n}(\mathcal{M})$ it follows that $d\left(\iota_{X} \omega\right)=\operatorname{div}_{\omega}(X) \omega$ for some number $\operatorname{div}_{\omega}(X)$.

Recall that $\operatorname{div}_{g}: \Gamma_{\mathscr{C} \infty}(T \mathcal{M}) \rightarrow \mathscr{C}^{\infty}(\mathcal{M})$ is the divergence operator defined by

$$
d\left(\iota_{X} \omega_{g}\right)=\operatorname{div}_{g}(X) \omega_{g} \text { for every } X \in \Gamma_{\mathscr{C}} \infty(T \mathcal{M}),
$$

where $\omega_{g} \in \Omega^{n}(\mathcal{M})$ denotes the volume element obtained from the metric $g$. In local coordinates $\left(x_{1}, \ldots, x_{n}\right)$, for $X=\sum_{j=1}^{n} b_{j} \frac{\partial}{\partial x_{j}} \in \Gamma_{\mathscr{C} \infty}(T \mathcal{M})$ divergence becomes

$$
\operatorname{div}_{g}(X)=\frac{1}{\sqrt{\operatorname{det} g}} \sum_{i=1}^{n} \frac{\partial}{\partial x_{i}}\left(b_{i} \sqrt{\operatorname{det} g}\right) .
$$

Note that if $X, Y \in \Gamma_{C^{\infty}}(T \mathcal{M})$ and $\omega \in \Omega^{n}(\mathcal{M})$, then $\iota_{X+Y} \omega=\iota_{X} \omega+\iota_{Y} \omega$. By this fact, we have $\operatorname{div}_{g}(X+Y)=\operatorname{div}_{g}(X)+\operatorname{div}_{g}(Y)$ Moreover, from (1) it follows that for $\varphi \in \mathscr{C}^{\infty}(M)$

$$
\operatorname{div}_{g}(\varphi X)=\varphi \operatorname{div}_{g} X+\left\langle\nabla_{g} \varphi, X\right\rangle_{g} .
$$

The Laplace operator $\Delta_{g}$ on smooth functions $\mathscr{C}^{\infty}(\mathcal{M})$ is defined by

$$
\Delta_{g}=-\operatorname{div}_{g} \circ \nabla_{g}
$$

is the Laplace-Beltrami operator on $(\mathcal{M}, g)$.

Note that for any $\varphi, \psi \in \mathscr{C}^{\infty}(\mathcal{M})$

$$
\begin{aligned}
& \Delta_{g}(\varphi+\psi)=\Delta_{g} \varphi+\Delta_{g} \psi, \\
& \Delta_{g}(\varphi \cdot \psi)=\psi \Delta_{g} \varphi+\varphi \Delta_{g} \psi-2\left\langle\nabla_{g} \varphi, \nabla_{g} \psi\right\rangle_{g} .
\end{aligned}
$$

In local coordinates $\left(x_{1}, \ldots, x_{n}\right)$, we have

$$
\Delta_{g}=-\frac{1}{\sqrt{\operatorname{det} g}} \sum_{i, j=1}^{n} \frac{\partial}{\partial x_{i}}\left(g^{i j} \sqrt{\operatorname{det} g} \frac{\partial}{\partial x_{j}}\right) .
$$

For example, let us consider the $n$-spere

$$
\mathbb{S}^{n}=\left\{\left(x_{1}, \ldots, x_{n+1}\right) \in \mathbb{R}^{n+1} ; x_{1}^{2}+\cdots+x_{n+1}^{2}=1\right\}
$$

in geodesic polar coordinates, to be more precise $\xi:(0, \pi)^{n-1} \times(0,2 \pi) \rightarrow \mathbb{S}^{n}$,

$$
\begin{aligned}
& x_{1}=\cos \theta_{1}, \\
& x_{2}=\sin \theta_{1} \cos \theta_{2}, \\
& x_{3}=\sin \theta_{1} \sin \theta_{2} \cos \theta_{3}, \\
& \vdots \\
& x_{n}=\sin \theta_{1} \sin \theta_{2} \cdots \cos \theta_{n}, \\
& x_{n+1}=\sin \theta_{1} \sin \theta_{2} \cdots \sin \theta_{n},
\end{aligned}
$$


where $0<\theta_{1}, \theta_{2}, \ldots, \theta_{n-1}<\pi, 0<\theta_{n}<2 \pi$. Then, we get

$$
\begin{gathered}
g_{\mathbb{S}^{n}}=\left[\begin{array}{cccccc}
1 & 0 & 0 & 0 & 0 & \cdots \cdots \\
0 & \sin ^{2} \theta_{1} & 0 & 0 & 0 & \cdots \cdots \\
0 & 0 & \sin ^{2} \theta_{1} \sin ^{2} \theta_{2} & 0 & 0 & \cdots \cdots \\
0 & 0 & 0 & \ddots & 0 & \cdots \cdots \\
\vdots & \vdots & \vdots & \vdots & \ddots & \cdots \cdots \\
0 & 0 & 0 & 0 & 0 & \sin ^{2} \theta_{1} \cdots \sin ^{2} \theta_{n-1}
\end{array}\right], \\
\sqrt{\operatorname{det} g_{\mathbb{S}^{n}}}=\prod_{\ell=1}^{n-1}\left(\sin \theta_{\ell}\right)^{n-\ell} .
\end{gathered}
$$

Moreover, the Laplace-Beltrami operator $\Delta_{\mathbb{S}^{n}}$ in these coordinates becomes

$$
-\frac{1}{\prod_{\ell=1}^{n-1}\left(\sin \theta_{\ell}\right)^{n-\ell}} \sum_{j=1}^{n} \frac{\partial}{\partial \theta_{j}}\left(a_{j}\left(\theta_{1}, \ldots, \theta_{n}\right) \frac{\partial}{\partial \theta_{j}}\right),
$$

where $a_{1}=1$ and for $j=2, \ldots, n, a_{j}=\frac{\prod_{\ell=1}^{n-1}\left(\sin \theta_{\ell}\right)^{n-\ell}}{\prod_{i=1}^{j-1} \sin ^{2} \theta_{i}}$.

We recall Stokes' Theorem and Divergence Theorem for manifolds.

Theorem 1. [Stokes' Theorem] Assume $\mathcal{M}$ is an oriented smooth compact n-manifold with boundary and $\alpha \in \Omega^{n-1}(\mathcal{M})$ have compact support. Denoting by $\iota: \partial \mathcal{M} \rightarrow \mathcal{M}$ the inclusion map, $\iota^{*} \alpha \in \Omega^{n-1}(\partial \mathcal{M})$. Then $\int_{\partial \mathcal{M}} \iota^{*} \alpha=\int_{\mathcal{M}} d \alpha$, or for short, $\int_{\partial \mathcal{M}} \alpha=\int_{\mathcal{M}} d \alpha$.

Theorem 2. [Divergence Theorem] Suppose $\mathcal{M}$ is a Riemannian manifold and $X$ is a $C^{1}$-vector field on $\mathcal{M}$. Then,

$$
\int_{\mathcal{M}} \operatorname{div}_{g}(X) d V_{g}=\int_{\partial \mathcal{M}}\langle X, \nu\rangle_{g} d \sigma_{g} .
$$

Here, $\operatorname{div}_{g}, d V_{g}$, and $\nu$ denote respectively the divergence operator on $(\mathcal{M}, g)$, the natural volume element on $(\mathcal{M}, g)$, and the unit vector normal to $\partial \mathcal{M}$.

From these results it follows

Theorem 3. [Green's Theorem] For a compact Riemannian manifold $(\mathcal{M}, g)$ with boundary $\partial \mathcal{M}$, if $\psi \in \mathscr{C}^{1}(\overline{\mathcal{M}})$ and $\varphi \in \mathscr{C}^{2}(\overline{\mathcal{M}})$, then the following equality is valid:

$$
\int_{\mathcal{M}} \psi \cdot \Delta_{\mathcal{M}} \phi d V_{g}=\int_{\mathcal{M}}\left\langle\nabla_{g} \psi, \nabla_{g} \phi\right\rangle d V_{g}-\int_{\partial \mathcal{M}} \psi \frac{\partial \phi}{\partial \nu} d \sigma_{g}
$$

Here, $\nabla_{g}$ denotes the gradient operator on the Riemannian manifold $(\mathcal{M}, g)$.

Green's Theorem yields

Theorem 4. [19] If $(\mathcal{M}, g)$ is a closed (i.e. compact without a boundary) Riemannian manifold, then

1 (Formal self-adjointness): $\left\langle\psi, \Delta_{\mathcal{M}} \phi\right\rangle_{\mathscr{L}_{2}\left(\mathcal{M}, d V_{g}\right)}=\left\langle\phi, \Delta_{\mathcal{M}} \psi\right\rangle_{\mathscr{L}_{2}\left(\mathcal{M}, d V_{g}\right)}$.

2 (Positivity): $\left\langle\Delta_{\mathcal{M}} \phi, \phi\right\rangle_{\mathscr{L}_{2}\left(\mathcal{M}, d V_{g}\right)} \geq 0$.

Here, $\mathscr{L}_{2}\left(\mathcal{M}, d V_{g}\right)$ is the Hilbert space

$$
\left\{f: \mathcal{M} \rightarrow \mathbb{R} ;\langle\phi, \phi\rangle_{\mathscr{L}_{2}\left(\mathcal{M}, d V_{g}\right)}:=\int_{\mathcal{M}} \phi^{2}(x) d V_{g}(x)<\infty\right\} .
$$


Recall that eigenvalues of the Laplacian on $n$-sphere $\mathbb{S}^{n} \subset \mathbb{R}^{n+1}$ are $\lambda_{\ell}=\ell(\ell+n-1), \ell=0,1,2, \ldots$ The corresponding eigenfunctions are restrictions of harmonic polynomials to the sphere.

\section{Elliptic differential equations on manifolds}

Neumann-Bitsadze-Samarskii type nonlocal boundary value problem on manifold

Let $\left(a_{i}, b_{i}\right) \subset(0, \pi), i=1, \ldots, n-1$ and $\left(a_{n}, b_{n}\right) \subset(0,2 \pi)$. We consider the domain

$$
\Omega=\xi\left(\left(a_{1}, b_{1}\right) \times \cdots \times\left(a_{n-1}, b_{n-1}\right) \times\left(a_{n}, b_{n}\right)\right) \subset \mathbb{S}^{n},
$$

where $\xi:(0, \pi)^{n-1} \times(0,2 \pi) \rightarrow \mathbb{S}^{n}$ is the geodesic polar parametrization (2).

$$
\left\{\begin{array}{l}
-u_{t t}(t, x)+\Delta_{\mathbb{S}^{n}} u(t, x)+\delta u(t, x)=f(t, x), \quad x \in \Omega, 0<t<1, \\
u_{t}(0, x)=0, \quad u_{t}(1, x)=\sum_{i=1}^{p} \beta_{i} u_{t}\left(\lambda_{i}, x\right), \quad x \in \Omega \\
\sum_{i=1}^{p}\left|\beta_{i}\right| \leq 1, \quad 0 \leq \lambda_{1}<\cdots<\lambda_{p}<1,\left.\quad \frac{\partial u}{\partial \vec{n}}(t, x)\right|_{x \in \partial \Omega}=0 .
\end{array}\right.
$$

Here, $\Delta_{\mathbb{S}^{n}}$ is the Laplace-Beltrami operator on the Riemannian manifold $\left(\mathbb{S}^{n}, g_{\mathbb{S}^{n}}\right)$ and $\delta>0$.

We prove

Theorem 5. For the solutions of problem (5), the following coercivity estimate holds:

$$
\left\|u_{t t}\right\|_{\mathscr{C}^{\alpha}\left(\mathscr{L}_{2}\left(\Omega, d V_{g}\right)\right)}+\|u\|_{\mathscr{C}^{\alpha}\left(\mathscr{W}_{2}^{2}\left(\Omega, d V_{g}\right)\right)} \leq \frac{K\left(\delta, \lambda_{p}\right)}{\alpha(1-\alpha)}\|f\|_{\mathscr{C}^{\alpha}\left(\mathscr{L}_{2}\left(\Omega, d V_{g}\right)\right)}
$$

Here, $K$ is independent of $f(t, x)$.

Let us consider Equation (5) as the following nonlocal boundary value problem of Bitsadze Samarskii type

$$
\left\{\begin{array}{l}
-U^{\prime \prime}(t)+\mathbf{L} U(t)=F(t), 0 \leq t \leq 1 \\
U_{t}(0)=0, U_{t}(1)=\sum_{i=1}^{p} \beta_{i} U_{t}\left(\lambda_{i}\right) \\
\sum_{i=1}^{p}\left|\beta_{i}\right| \leq 1,0 \leq \lambda_{1}<\cdots<\lambda_{p}<1
\end{array}\right.
$$

in $\mathscr{L}_{2}\left(\Omega, d V_{g}\right)$ with the self adjoint and positive definite operator $\mathbf{L}=\Delta_{\mathbb{S}^{n}}+\delta I$. Here, $I$ denotes the identity operator.

The proof of Theorem 5 is based on the symmetry property of $\mathbf{L}$, Theorem 6 with $H=\mathscr{L}_{2}\left(\Omega, d V_{g}\right)$ and Theorem 7 on the coercivity inequality for the solution of elliptic differential problem in $\mathscr{L}_{2}\left(\Omega, d V_{g}\right)$. Theorem 6. [17] Let $A$ be a self-adjoint positive definite operator with dense domain $D(A)$ in a Hilbert space H. Let $\varphi, \psi \in E_{\alpha}\left(D\left(A^{1 / 2}\right), H\right)$. Then the following elliptic type differential problem

$$
\left\{\begin{array}{l}
-v_{t t}(t, x)+A v(t)=g(t), \quad 0<t<1 \\
v_{t}(0)=\varphi, \quad v_{t}(1)=\sum_{i=1}^{p} \beta_{i} v_{t}\left(\lambda_{i}\right)+\psi \\
\sum_{i=1}^{p}\left|\beta_{i}\right| \leq 1, \quad 0 \leq \lambda_{1}<\cdots<\lambda_{p}<1
\end{array}\right.
$$


is well-posed in Hölder space $\mathscr{C}^{\alpha}(H)$ and for the solutions of (6) the following coercivity inequality holds:

$$
\left\|v^{\prime \prime}\right\|_{\mathscr{C}^{\alpha}(H)}+\|A v\|_{\mathscr{C}^{\alpha}(H)} \leq K(\delta)\left[\left\|A^{1 / 2} \varphi\right\|_{H}+\left\|A^{1 / 2} \psi\right\|_{H}\right]+\frac{K\left(\delta, \lambda_{p}\right)}{\alpha(1-\alpha)}\|g\|_{\mathscr{C}^{\alpha}(H)} .
$$

Theorem \%. The solutions of the following elliptic differential problem

$$
\left\{\begin{array}{l}
\Delta_{\mathbb{S}^{n}} u(\xi(\vec{\theta}))=\omega(\xi(\vec{\theta})), \vec{\theta}=\left(\theta_{1}, \ldots, \theta_{n}\right) \in\left(a_{1}, b_{1}\right) \times \cdots \times\left(a_{n}, b_{n}\right), \\
\frac{\partial u(\xi(\vec{\theta})}{\partial \vec{n}}=0, \vec{\theta} \text { in boundary of }\left[a_{1}, b_{1}\right] \times \cdots \times\left[a_{n}, b_{n}\right]
\end{array}\right.
$$

satisfy the coercivity inequality

$$
\sum_{i=1}^{n}\left\|u_{\theta_{i} \theta_{i}}\right\|_{\mathscr{L}_{2}\left(\Omega, d V_{g}\right)} \leq K_{1}\|\omega\|_{\mathscr{L}_{2}\left(\Omega, d V_{g}\right)} .
$$

The proof of Theorem 7 is based on the following theorem.

Theorem 8. [8] For the solutions of the elliptic differential problem

$$
\left\{\begin{array}{l}
A^{\xi} u(\xi)=\omega(\xi), \quad \xi \in\left(\alpha_{1}, \beta_{1}\right) \times \cdots \times\left(\alpha_{n}, \beta_{n}\right) \\
\frac{\partial u(\xi)}{\partial \vec{n}}=0, \quad \xi \text { in boundary }\left[\alpha_{1}, \beta_{1}\right] \times \cdots \times\left[\alpha_{n}, \beta_{n}\right]
\end{array}\right.
$$

the following coercivity inequality

$$
\sum_{i=1}^{n}\left\|u_{\xi_{i} \xi_{i}}\right\|_{\mathscr{L}_{2}\left(\left(\alpha_{1}, \beta_{1}\right) \times \cdots \times\left(\alpha_{n}, \beta_{n}\right)\right)} \leq K_{2}\|\omega\|_{\mathscr{L}_{2}\left(\left(\alpha_{1}, \beta_{1}\right) \times \cdots \times\left(\alpha_{n}, \beta_{n}\right)\right)}
$$

is valid. Here, $A^{\xi}=\sum_{r=1}^{n} \frac{\partial}{\partial \xi_{r}}\left(a_{r}(\xi) \frac{\partial}{\partial \xi_{r}}\right)$ and $a_{r}(\xi) \geq a>0, r=1, \ldots, n$.

Proof of Theorem 7 . Clearly, the image $\xi(\vec{\theta})$ of boundary of the $n$-cube $\left[a_{1}, b_{1}\right] \times \cdots \times\left[a_{n}, b_{n}\right]$ is the boundary of $\Omega$. This parametrization maps $\left(a_{1}, b_{1}\right) \times \cdots \times\left(a_{n}, b_{n}\right)$ to the interior of $\Omega$. Let $u: \Omega \rightarrow \mathbb{R}$ be so that $\frac{\partial u}{\partial \nu}$ vanishes on the boundary of $\Omega$. Then, $v=u \circ \xi:\left[a_{1}, b_{1}\right] \times \cdots \times\left[a_{n}, b_{n}\right] \rightarrow \mathbb{R}$ and $\frac{\partial v}{\partial \nu}$ vanishes on the boundary of the cube $\left[a_{1}, b_{1}\right] \times \cdots \times\left[a_{n}, b_{n}\right]$. Here, $\nu$ is the outward unit normal to the boundary.

For some constants $k, K>0$, on $\Omega$ we have $0<k \leq \prod_{\ell=1}^{n-1}\left(\sin \theta_{\ell}\right)^{n-\ell} \leq K$.

Equation (3) and Theorem 8 yield

$$
\begin{aligned}
\int_{\Omega}\left|\Delta_{\mathbb{S}^{n}} u(x)\right|^{2} d V_{g}(x) & =\int_{a_{1}}^{b_{1}} \cdots \int_{a_{n}}^{b_{n}} \frac{\left\{\sum_{j=1}^{n} \frac{\partial}{\partial \theta_{j}}\left(a_{j}(\vec{\theta}) \frac{\partial u \circ \xi(\vec{\theta})}{\partial \theta_{j}}\right)\right\}^{2}}{\prod_{\ell=1}^{n-1}\left(\sin \theta_{\ell}\right)^{n-\ell}} d \theta_{n} \cdots d \theta_{1} \\
& \geq \frac{1}{K} \int_{a_{1}}^{b_{1}} \cdots \int_{a_{n}}^{b_{n}}\left\{\sum_{j=1}^{n} \frac{\partial}{\partial \theta_{j}}\left(a_{j}(\vec{\theta}) \frac{\partial u \circ \xi(\vec{\theta})}{\partial \theta_{j}}\right)\right\}^{2} d \theta_{n} \cdots d \theta_{1} \\
& =\frac{1}{K}\left\|A^{\left(\theta_{1}, \ldots, \theta_{n}\right)} u \circ \xi\right\|_{\mathscr{L}_{2}\left(\left(a_{1}, b_{1}\right) \times \cdots \times\left(a_{n}, b_{n}\right)\right)}^{2} \\
& =\frac{1}{K}\left\|A^{\left(\theta_{1}, \ldots, \theta_{n}\right)} v\right\|_{\mathscr{L}_{2}\left(\left(a_{1}, b_{1}\right) \times \cdots \times\left(a_{n}, b_{n}\right)\right)}^{2} \\
& \geq \frac{1}{K \cdot K_{2}^{2}}\left(\sum_{i=1}^{n}\left\|v_{\theta_{i} \theta_{i}}\right\|_{\mathscr{L}_{2}\left(\left(a_{1}, b_{1}\right) \times \cdots \times\left(a_{n}, b_{n}\right)\right)}\right)^{2} .
\end{aligned}
$$


Hence, we obtain

$$
\left(\int_{\Omega}\left|\Delta_{\mathbb{S}^{n}} u(x)\right|^{2} d V_{g}(x)\right)^{1 / 2} \geq \frac{1}{\sqrt{K} K_{2}} \sum_{i=1}^{n}\left\|v_{\theta_{i} \theta_{i}}\right\|_{\mathscr{L}_{2}\left(\left(a_{1}, b_{1}\right) \times \cdots \times\left(a_{n}, b_{n}\right)\right)} .
$$

For $i=1, \ldots, n$, we have

$$
\begin{aligned}
\left\|v_{\theta_{i} \theta_{i}}\right\|_{\mathscr{L}_{2}\left(\left(a_{1}, b_{1}\right) \times \cdots \times\left(a_{n}, b_{n}\right)\right)} & =\left(\int_{a_{1}}^{b_{1}} \ldots \int_{a_{n}}^{b_{n}}\left|v_{\theta_{i} \theta_{i}}\left(\theta_{1}, \ldots, \theta_{n}\right)\right|^{2} d \theta_{n} \cdots d \theta_{1}\right)^{1 / 2} \\
& \geq\left(\int_{a_{1}}^{b_{1}} \ldots \int_{a_{n}}^{b_{n}}\left|v_{\theta_{i} \theta_{i}}\left(\theta_{1}, \ldots, \theta_{n}\right)\right|^{2} \frac{\prod_{\ell=1}^{n-1}\left(\sin \theta_{\ell}\right)^{n-\ell}}{K} d \theta_{n} \cdots d \theta_{1}\right)^{1 / 2} \\
& =\frac{1}{\sqrt{K}}\left(\int_{a_{1}}^{b_{1}} \ldots \int_{a_{n}}^{b_{1}}\left|v_{\theta_{i} \theta_{i}}\left(\theta_{1}, \ldots, \theta_{n}\right)\right|^{2} \prod_{\ell=1}^{n-1}\left(\sin \theta_{\ell}\right)^{n-\ell} d \theta_{n} \cdots d \theta_{1}\right)^{1 / 2} \\
& =\frac{1}{\sqrt{K}}\left(\int_{a_{1}}^{b_{1}} \ldots \int_{a_{n}}^{b_{n}}\left|(u \circ \xi)_{\theta_{i} \theta_{i}}\left(\theta_{1}, \ldots, \theta_{n}\right)\right|^{2} \prod_{\ell=1}^{n-1}\left(\sin \theta_{\ell}\right)^{n-\ell} d \theta_{n} \cdots d \theta_{1}\right)^{1 / 2} \\
& =\frac{1}{\sqrt{K}}\left\|u_{\theta_{i} \theta_{i}}\right\|_{\mathscr{L}_{2}\left(\Omega, d V_{g}\right)} .
\end{aligned}
$$

Combining equations (7) and (8), we get

$$
\left(\int_{\Omega}\left|\Delta_{\mathbb{S}_{n}} u(x)\right|^{2} d V_{g}(x)\right)^{1 / 2} \geq \frac{1}{K \cdot K_{2}} \sum_{i=1}^{n}\left\|u_{\theta_{i} \theta_{i}}\right\|_{\mathscr{L}_{2}\left(\Omega, d V_{g}\right)} .
$$

This is the end of the proof of Theorem 7 .

\section{Dirichlet-Bitsadze-Samarskii type nonlocal boundary value problem on manifold}

Assume $(\mathcal{M}, g)$ is a closed orientable Riemannian manifold (such as $n$-sphere $\mathbb{S}^{n}, n$-torus $\mathbb{T}^{n}$ ). Let us consider the mixed boundary value problem of Dirichlet-Bitsadze-Samarskii type

$$
\left\{\begin{array}{l}
-u_{t t}(t, x)+\Delta_{\mathcal{M}} u(t, x)+\delta u(t, x)=f(t, x), \quad x \in \mathcal{M}, \quad 0<t<1, \\
u(0, x)=\varphi(x), u(1, x)=\sum_{j=1}^{p} \alpha_{j} u\left(\lambda_{j}, x\right)+\psi(x), \quad x \in \mathcal{M}, \\
0<\lambda_{1}<\cdots<\lambda_{p}<1, \quad \sum_{j=1}^{p}\left|\alpha_{j}\right| \leq 1
\end{array}\right.
$$

where $\Delta_{\mathcal{M}}$ is the Laplace-Beltrami operator on the Riemannian manifold $(\mathcal{M}, g)$.

We prove 
Theorem 9. If $\varphi, \psi \in D(\mathbf{L})$, then for the solution of (9) we have the following coercivity inequality

$$
\begin{aligned}
& \left\|u_{t t}\right\|_{\mathscr{C}_{01}^{\alpha}\left(\mathscr{L}_{2}\left(\mathcal{M}, d V_{g}\right)\right)}+\|\mathbf{L} u\|_{\mathscr{C}_{01}^{\alpha}\left(\mathscr{L}_{2}\left(\mathcal{M}, d V_{g}\right)\right)} \\
& \leq K\left[\|\mathbf{L} \varphi\|_{\mathscr{L}_{2}\left(\mathcal{M}, d V_{g}\right)}+\|\mathbf{L} \psi\|_{\mathscr{L}_{2}\left(\mathcal{M}, d V_{g}\right)}\right]+\frac{K\left(\delta, \lambda_{1}, \lambda_{p}\right)}{\alpha(1-\alpha)}\|f\|_{\mathscr{C}_{01}^{\alpha}\left(\mathscr{L}_{2}\left(\mathcal{M}, d V_{g}\right)\right)} .
\end{aligned}
$$

Here, $K\left(\delta, \lambda_{1}, \lambda_{p}\right)$ does not depend on $\varphi(x), \psi(x)$, and $f(t, x)$.

Let us consider problem (9) as the following nonlocal boundary value problem of Bitsadze Samarskii type

$$
\left\{\begin{array}{l}
-U^{\prime \prime}(t)+\mathbf{L} U(t)=F(t), \quad t \in(0,1) \\
U(0)=\varphi, \quad U(1)=\sum_{j=1}^{p} \alpha_{j} U\left(\lambda_{j}\right)+\psi \\
0<\lambda_{1}<\cdots<\lambda_{p}<1, \quad \sum_{j=1}^{p}\left|\alpha_{j}\right| \leq 1
\end{array}\right.
$$

in $\mathscr{L}_{2}\left(\mathcal{M}, d V_{g}\right)$ with the self-adjoint and positive definite operator $\mathbf{L}=\Delta_{\mathcal{M}}+\delta I$. Here, $I$ denotes the identity operator, $\|U\|_{\mathscr{L}_{2}\left(\mathcal{M}, d V_{g}\right)}=\left(\int_{\mathcal{M}} U^{2}(x) d V_{g}(x)\right)^{1 / 2}$, and $d V_{g}$ denotes natural volume element of $\mathcal{M}$ obtained from metric tensor $g$.

The proof of Theorem 9 relies on the following theorem.

Theorem 10. [16] Assume $A$ is a self-adjoint positive definite operator with dense $D(A) \subset H$ in a Hilbert space $H$ and $\varphi, \psi \in D(A)$. Then, the following boundary value problem

$$
\left\{\begin{array}{c}
-v_{t t}(t, x)+A v(t)=f(t), \quad 0<t<1, \\
v(0)=\varphi, \quad v(1)=\sum_{j=1}^{p} \alpha_{j} v\left(\lambda_{j}\right)+\psi \\
0<\lambda_{1}<\cdots<\lambda_{p}<1, \quad \sum_{j=1}^{p}\left|\alpha_{j}\right| \leq 1
\end{array}\right.
$$

is well-posed in Hölder space $\mathscr{C}_{01}^{\alpha}(H)$. Moreover, the solutions of the problem satisfy the following coercivity inequality

$$
\left\|v^{\prime \prime}\right\|_{\mathscr{C}_{01}^{\alpha}(H)}+\|A v\|_{\mathscr{C}_{01}^{\alpha}(H)} \leq K\left[\|A \varphi\|_{H}+\|A \psi\|_{H}\right]+\frac{K\left(\delta, \lambda_{1}, \lambda_{p}\right)}{\alpha(1-\alpha)}\|f\|_{\mathscr{C}_{01}^{\alpha}(, H)} .
$$

Here, $K\left(\delta, \lambda_{1}, \lambda_{p}\right)$ is independent of of $\varphi(x), \psi(x)$, and $f(t, x) . \mathscr{C}_{01}^{\alpha}(H)(0<\alpha<1)$ denotes the Banach space which is the completion of of smooth funtions $v:[0,1] \rightarrow H$ with the following norm

$$
\|v\|_{\mathscr{C}_{01}^{\alpha}(H)}=\|v\|_{\mathscr{C}(H)}+\sup _{0 \leq t<t+\tau \leq 1} \frac{(1-t)^{\alpha}(t+\tau)^{\alpha}\|v(t+\tau)-v(t)\|_{H}}{\tau^{\alpha}}
$$

and $\|v\|_{\mathscr{C}(H)}=\max _{0 \leq t \leq 1}\|v(t)\|_{H}$ 
Dirichlet-Bitsadze-Samarskii type nonlocal boundary value problem on a relatively compact domain

For the domain $\Omega$ in (4), let us consider the Dirichlet-Bitsadze-Samarskii type mixed boundary value problem

$$
\left\{\begin{array}{l}
-u_{t t}(t, x)+\Delta_{\mathbb{S}^{n}} u(t, x)=f(t, x), \quad x \in \Omega, \quad t \in(0,1), \\
u(0, x)=\varphi(x), u(1, x)=\sum_{j=1}^{p} \alpha_{j} u\left(\lambda_{j}, x\right)+\psi(x), \quad x \in \Omega, \\
0<\lambda_{1}<\cdots<\lambda_{p}<1, \quad \sum_{j=1}^{p}\left|\alpha_{j}\right| \leq 1, \\
u(t, x)=0, \quad x \in \partial \Omega,
\end{array}\right.
$$

where $\Delta_{\mathbb{S}^{n}}$ is the Laplace-Beltrami operator on the Riemannian manifold $\left(\mathbb{S}^{n}, g_{\mathbb{S}^{n}}\right)$.

We have

Theorem 11. The solutions of nonlocal boundary value problem (11) satisfy following coercivity inequality

$$
\begin{aligned}
\left\|u_{t t}\right\|_{\mathscr{C}_{01}^{\alpha}\left(\mathscr{L}_{2}\left(\Omega, d V_{g}\right)\right)}+\|u\|_{\mathscr{C}_{01}^{\alpha}\left(\mathscr{W}_{2}^{2}\left(\Omega, d V_{g}\right)\right)} \leq & K\left[\|\varphi\|_{\mathscr{W}_{2}^{2}\left(\Omega, d V_{g}\right)}+\|\psi\|_{\mathscr{W}_{2}^{2}\left(\Omega, d V_{g}\right)}\right] \\
& +\frac{K\left(\delta, \lambda_{1}, \lambda_{p}\right)}{\alpha(1-\alpha)}\|f\|_{\mathscr{C}_{01}^{\alpha}\left(\mathscr{L}_{2}\left(\Omega, d V_{g}\right)\right)}
\end{aligned}
$$

where $K\left(\delta, \lambda_{1}, \lambda_{p}\right)$ does not depend on $\varphi(x), \psi(x)$, and $f(t, x)$.

Let us consider problem (11) as the nonlocal boundary value problem (10) in the Hilbert space $H=\mathscr{L}_{2}\left(\Omega, d V_{g}\right)$ with the self-adjoint positive definite operator $\mathbf{L}=\Delta_{\mathbb{S}^{n}}$.

The proof of Theorem 11 is based on the symmetry properties of the operator $\mathbf{L}$ defined by formula (11), Theorem 10 with $H=\mathscr{L}_{2}\left(\Omega, d V_{g}\right)$, and the following result which is about the coercivity estimate for the solution of the elliptic type differential equation in $\mathscr{L}_{2}\left(\Omega, d V_{g}\right)$.

Theorem 12. For the following differential equation of elliptic type

$$
\left\{\begin{array}{l}
\Delta_{\mathbb{S}^{n}} u(\xi(\vec{\theta}))=\omega(\xi(\vec{\theta})), \vec{\theta}=\left(\theta_{1}, \ldots, \theta_{n}\right) \in\left(a_{1}, b_{1}\right) \times \cdots \times\left(a_{n}, b_{n}\right), \\
u\left(\xi(\vec{\theta})=0, \vec{\theta} \text { in boundary of }\left[a_{1}, b_{1}\right] \times \cdots \times\left[a_{n}, b_{n}\right]\right.
\end{array}\right.
$$

we have the following coercivity estimate

$$
\sum_{i=1}^{n}\left\|u_{\theta_{i} \theta_{i}}\right\|_{\mathscr{L}_{2}\left(\Omega, d V_{g}\right)} \leq K_{1}\|\omega\|_{\mathscr{L}_{2}\left(\Omega, d V_{g}\right)}
$$

The proof of Theorem 12 relies on the following theorem.

Theorem 13. [8] For the solutions of the elliptic differential problem

$$
\left\{\begin{array}{l}
A^{\xi} u(\xi)=\omega(\xi), \quad \xi \in\left(\alpha_{1}, \beta_{1}\right) \times \cdots \times\left(\alpha_{n}, \beta_{n}\right), \\
u(\xi)=0, \xi \text { in boundary }\left[\alpha_{1}, \beta_{1}\right] \times \cdots \times\left[\alpha_{n}, \beta_{n}\right]
\end{array}\right.
$$

the coercivity inequality

$$
\sum_{r=1}^{n}\left\|u_{\xi_{r} \xi_{r}}\right\|_{\mathscr{L}_{2}\left(\left(\alpha_{1}, \beta_{1}\right) \times \cdots \times\left(\alpha_{n}, \beta_{n}\right)\right)} \leq K_{2} \mid \omega \|_{\mathscr{L}_{2}\left(\left(\alpha_{1}, \beta_{1}\right) \times \cdots \times\left(\alpha_{n}, \beta_{n}\right)\right)}
$$


is valid. Here, $A^{\xi}=\sum_{r=1}^{n} \frac{\partial}{\partial \xi_{r}}\left(a_{r}(\xi) \frac{\partial}{\partial \xi_{r}}\right)$ and $a_{r}(\xi) \geq a>0, r=1, \ldots, n$.

\section{Acknowledgement}

The publication has been prepared with the support of the "RUDN University Program 5-100"

\section{References}

1 Ladyzhenskaya O.A. Linear and quasilinear equations of parabolic type / O.A. Ladyzhenskaya, V.A. Solonnikov, N.N. Ural'tseva. - Rhode Island: American Mathematical Society, 1968.

2 Ladyzhenskaya O.A. Linear and quasilinear elliptic equations / O.A. Ladyzhenskaya, N.N. Ural'tseva. - New York: Academic Press, 1968.

3 Вишик М.Я. Дифференциальные уравнения с частными производными / М.Я. Вишик, А.Д. Мышкис, О.А. Олейник // Математика СССР за сорок лет, 1917-1957: [В 2 т.] Т. 1 / Под ред. А.Г. Куроша (глав.ред.), В.И. Битюцкова, В.Г. Болтянского, Е.Б. Дынкина, Г.Е. Шилова, А.П. Юшкевича. - М: ГИФМЛ, 1959. - С. 563-636.

4 Крейн С.Г. Линейные дифференциальные уравнения в банаховом пространстве / С.Г. Крейн. - М.: Наука, 1966.

5 Bitsadze, A.V. Some elementary generalizations of linear elliptic boundary value problems / A.V. Bitsadze, A.A. Samarskii // Doklady Akademii Nauk SSSR. - 1969. - 185. No. 4. P. 739-740.

6 Sobolevskii P.E. On elliptic equations in a Banach space / P.E. Sobolevskii // Differentsial'nye Uravneniya. - 1969. - 4. - No. 7. - P. 1346-1348.

7 Sobolevskii P.E. The coercive solvability of difference equations / P.E. Sobolevskii // Doklady Akademii Nauk SSSR. - 1971. - 201. - No.5. - P. 1063-1066.

8 Sobolevskii P.E. Some properties of the solutions of differential equations in fractional spaces / P.E. Sobolevskii // in Trudy Nauchno issledovatelskoho instituta. Matematicheski Voronezhskoho Hosudarstvennoho universiteta. - 1975. - 14. - P. 68-74.

9 Sobolevskii P.E. Well-posedness of difference elliptic equation / P.E. Sobolevskii // Discrete Dynamics in Nature and Society - 1997. - 1. - No.3. - P. 219-231.

10 Gershteyn L.M. Well-posedness of the general boundary value problem for the second order elliptic equations in a Banach space / L.M. Gershteyn, \& P.E. Sobolevskii // Differentsial'nye Uravneniya. - 1975. - 11. - No.7. - P. 1335-1337.

11 Kapanadze D.V. On the Bitsadze-Samarskii nonlocal boundary value problem / D.V. Kapanadze // Journal of Differential Equation. - 1987. - 23. - No.3. - P. 543-545.

12 Clement $\mathrm{Ph}$. On the regularity of abstract Cauchy problems and boundary value problems / Ph. Clement, S. Guerre-Delabrire // Atti della Accademia Nazionale dei Lincei. Classe di Scienze Fisiche, Matematiche e Naturali. Rendiconti Lincei. Serie IX. Matematica e Applicazioni. - 1998. - 9. - No. 4. - P. 245-266.

13 Ashyralyev A. A note on the Bitsadze-Samarskii type nonlocal boundary value problem in a Banach space / A. Ashyralyev // J.Math. Anal.Appl. - 2008. - 344. - P. 557-573.

14 Ashyralyev A. Well-posedness of the elliptic equations in a space of smooth functions / A. Ashyralyev // Boundary Value Problems. - 1989. - 2. No. 2. - P. 82-86.

15 Ashyralyev A. On well-posedness of the nonlocal boundary for elliptic equations / A. Ashyralyev // Numer. Funct. Anal. Optim. - 2003. - 4. - P. 1-15. 
16 Ashyralyev A. On Bitsadze-Samarskii type nonlocal boundary value problems for elliptic differential and difference equations: well-posedness / A. Ashyralyev, E. Ozturk // Applied Mathematics and Computation. - 2012. - 219. - P. 1093-1107.

17 Ashyralyev A. A note on Bitsadze-Samarskii type nonlocal boundary problems: well-posednesss / A. Ashyralyev, F.S.O. Tetikoglu // Numer. Funct. Anal. Optim. - 2013. - 34. - P. 939-975.

18 Ashyralyev A. On well-posedness of difference schemes for abstract elliptic problems in $L_{p}([0 ; 1] ; E)$ spaces / A. Ashyralyev, C. Cuevas, S. Piskarev // Numer. Funct. Anal. Optim. 2008. - 29. - P. 43-65.

19 Chavel I. Eigenvalues in Riemannian geometry / I. Chavel. - New York: Academic Press, 1984.

20 Urakawa H. Geometry of Laplace-Beltrami operator on a complete Riemannian manifold / H. Urakawa // Advanced Studies in Pure Mathematics. - 1993. - 22. - P. 347-406.

А. Ашыралыев, Я. Созен, Ф. Незенжи

\title{
Көпбейнедегі эллипстік дифференциалдық теңдеу туралы ескерту
}

\begin{abstract}
Евклидтік кеңістігінде бейлокальді типті эллипстік шеттік есептері үшін қойылған есептің корректілігі бірнеше авторлармен жақсы және толық зерттелген. Басқа жағынан, осы мәселелер көпбейнеде зерттелмеген. Мақалада тегіс тұйық көпбейнеде дифференциалдық теңдеу қарастырылған. Эллипстік типті бейлокальді шеттік есептің корректілігі қойылады, нақтырақ айтатын болсақ көпбейнеде, Гольдер кеңістігіндегі көпбейнеде Дирихле-Бицадзе-Самарский түріндегі бейлокальді шеттік есебі. Сонымен қатар, әртүрлі Гольдер нормасында тегіс көпбейнеде бейлокальді типті эллипстік шеттік есебін шығару үшін мәжбүрлі жаңа теңсіздіктер анықталған.
\end{abstract}

Kiлm сөздер: көпбейнедегі дифференциалдық теңдеу, корректілігі, өзіне-өзі түйіндес оң анықталған оператор.

\author{
А. Ашыралыев, Я. Созен, Ф. Незенжи
}

\section{Замечание об эллиптических дифференциальных уравнениях на многообразии}

\begin{abstract}
Для эллиптических краевых задач нелокального типа в евклидовом пространстве корректность поставленной задачи была хорошо изучена несколькими авторами. С другой стороны, такие проблемы на многообразиях широко не изучены. В настоящей статье рассмотрены дифференциальные уравнения на гладких замкнутых многообразиях. Установлена корректность нелокальных краевых задач эллиптического типа, а именно нелокальной краевой задачи типа Неймана-Бицадзе-Самарского на многообразиях, а также нелокальной краевой задачи типа Дирихле-Бицадзе-Самарского на многообразиях в пространствах Гольдера. Кроме того, в различных нормах Гольдера установлены новые неравенства коэрцитивности для решений краевых задач эллиптического нелокального типа на гладких многообразиях.
\end{abstract}

Ключевые слова: дифференциальные уравнения на многообразиях, корректность, самосопряженный положительно определенный оператор. 


\section{References}

1 Ladyzhenskaya, O.A., Solonnikov, V.A., \& Ural'tseva, N.N. (1968). Linear and quasilinear equations of parabolic type. Rhode Island: American Mathematical Society.

2 Ladyzhenskaya, O.A., \& Ural'tseva, N.N. (1968). Linear and Quasilinear Elliptic Equations. New York: Academic Press.

3 Vishik, M.L., Myshkis, A.D., \& Oleinik, O.A. (1959). Differentsialnye uravneniia s chastnymi proizvodnymi [Partial differential equations in: mathematics in USSR in the last 40 years]. Matematika SSSR za sorok let, 1917-1957. (Vol. 1-2; Vol. 2). Moscow: Fizmathiz [in Russian].

4 Krein, S.G. (1966). Lineinye differensialnye uravneniia $v$ banakhovom prostranstve /Linear Differential Equations in Banach Space]. Moscow: Nauka [in Russian].

5 Bitsadze, A.V., \& Samarskii, A.A. (1969). Some elementary generalizations of linear elliptic boundary value problems. Doklady Akademii Nauk SSSR, 185,4, 739-740.

6 Sobolevskii, P.E. (1969). On elliptic equations in a Banach space. Differentsial'nye Uravneniya, 4, 7, 1346-1348.

7 Sobolevskii, P.E. (1971). The coercive solvability of difference equations. Doklady Akademii Nauk SSSR, 201, 5, 1063-1066.

8 Sobolevskii, P.E. (1975). Some properties of the solutions of differential equations in fractional spaces. in: Trudy Nauchn. -Issled. Inst. Mat. Voronezh. Gos. Univ. 14, 68-74.

9 Sobolevskii, P.E. (1997). Well-posedness of difference elliptic equation. Discrete Dynamics in Nature and Society 1, 3, 219-231.

10 Gershteyn, L.M.,\& Sobolevskii, P.E. (1975). Well-posedness of the general boundary value problem for the second order elliptic equations in a Banach space. Differentsial'nye Uravneniya, 11, 7, $1335-1337$.

11 Kapanadze, D.V. (1987). On the Bitsadze-Samarskii nonlocal boundary value problem. Journal of Differential Equation, 23, 3, 543-545.

12 Clement, Ph.,\& Guerre-Delabrire, S. (1998). On the regularity of abstract Cauchy problems and boundary value problems. Atti della Accademia Nazionale dei Lincei. Classe di Scienze Fisiche, Matematiche e Naturali. Rendiconti Lincei. Serie IX. Matematica e Applicazioni, 9, 4, 245-266.

13 Ashyralyev, A. (2008). A note on the Bitsadze-Samarskii type nonlocal boundary value problem in a Banach space. Journal of Mathematical Analysis and Applications, 344, 1, 557-573.

14 Ashyralyev, A. (1989). Well-posedness of the elliptic equations in a space of smooth functions. Boundary Value Problems, 2, 2, 82-86.

15 Ashyralyev, A. (2003). On well-posedness of the nonlocal boundary value problem for elliptic equations. Numerical Functional Analysis and Optimization, 24, 1-2, 1-15.

16 Ashyralyev, A., \& Ozturk, E. (2012). On Bitsadze-Samarskii type nonlocal boundary value problems for elliptic differential and difference equations: well-posedness. Applied Mathematics and Computation, 219, 1093-1107.

17 Ashyralyev, A., \& Ozesenli Tetikoglu, F. (2013). A note on Bitsadze-Samarskii type nonlocal boundary value problems: well-posedness. Numerical Functional Analysis and Optimization, 34,9, 939-975.

18 Ashyralyev, A., Cuevas, C., \& Piskarev, S. (2008). On well-posedness of difference schemes for abstract elliptic problems in $L_{p}([0 ; 1] ; E)$ spaces. Numerical Functional Analysis and Optimization, 29, 43-65.

19 Chavel, I. (1984). Eigenvalues in Riemannian geometry. New York: Academic Press.

20 Urakawa, H. (1993). Geometry of Laplace-Beltrami operator on a complete Riemannian manifold. Advanced Studies in Pure Mathematics, 22, 347-406. 